Relations industrielles

Industrial Relations

\title{
White-and-Blue-Collars in a Mill Shutdown, by Felcian F. Foltman, New York State School for Industrial and Labor Relations, Cornell University, 1968, 132 pages.
}

\section{L.-René Parenteau}

\section{Volume 23, numéro 4, 1968}

URI : https://id.erudit.org/iderudit/027975ar

DOI : https://doi.org/10.7202/027975ar

Aller au sommaire du numéro

Éditeur(s)

Département des relations industrielles de l'Université Laval

ISSN

0034-379X (imprimé)

1703-8138 (numérique)

Découvrir la revue

Citer ce compte rendu

Parenteau, L.-R. (1968). Compte rendu de [White-and-Blue-Collars in a Mill Shutdown, by Felcian F. Foltman, New York State School for Industrial and Labor Relations, Cornell University, 1968, 132 pages.] Relations industrielles / Industrial Relations, 23(4), 707-707. https://doi.org/10.7202/027975ar

Tous droits réservés @ C Département des relations industrielles de l'Université Laval, 1968
Ce document est protégé par la loi sur le droit d'auteur. L'utilisation des services d’Érudit (y compris la reproduction) est assujettie à sa politique d'utilisation que vous pouvez consulter en ligne.

https://apropos.erudit.org/fr/usagers/politique-dutilisation/ 
Dans ce livre, les auteurs du Managerial Grid voient dans le conflit trois dimensions de base: la première « The Win-Lose Orientation to Intergroup Disagreement * suppose pour que l'un gagne que l'autre perde. En second lieu, les auteurs examinent la solution d'un règlement de conflit par une tierce personne, pour finalement en arriver à l'approche de la recherche de la solution en groupe.

Ce n'est donc pas un livre de théorie et de principes non vérifiés. Cependant, il ne fout pas le considérer comme un livre de recettes répondant à tous les problèmes de conflit entre les différents groupes d'une organisation.

Ce conflit, il est inévitable. II peut cependant être atténué dans une certaine mesure por l'application des sciences du comportement.

\section{Jean SEXTON}

White-and-Blue-Collors in a Mill Shutdown, by Felician F. Foltman, New York State School of Industrial and Labor Relations, Cornell University 1968, 132 pages.

La fermeture d'une usine ou la réduction des opérations mènent infailliblement au renvoi des plusieurs centaines de travailleurs. Le phénomène se présente de plus en plus fréquemment dans notre économie rapidement changeante; il est la source de situations tragiques pour les travailleurs concernés et soulève d'importants problèmes connus par toutes nos politiques de maind'oeuvre.

M. Foltman a entrepris l'étude d'un cas courant, mais il en fait une analyse qui peut servir dans la moyenne générale des cas. Son optique est profondément humaine car c'est en termes d'attitudes ouvrières qu'il analyse le problème: attitudes face à lo fermeture de l'usine, attitudes face au renvoi, à la mobilité exigée par ce renvoi, à la recherche d'un nouvel emploi. En plus de nous donner cette approche particulière du problème, $M$. Foltman nous fournit un modèle d'analyse.

$\mathrm{Ce}$ modèle d'analyse se retrouve essentiellement dans le questionnaire qu'il a utilisé pour son étude, dans l'approche globale du problème (sources et conséquences proches et éloignées), dans la description de variables propres et dans les relations (corrélations) qu'il a tenté d'établir entre ces variables pour dégager les conclusions généroles. Ainsi, por exemple, il étudie les relations entre l'âge, la formation et la mobilité des travailleurs; les relations entre le statut familial, la vie sociale, et la mobilité.

Si les résultats de l'analyse ne nous indiquent pas des solutions certaines à ce genre de problèmes, ils nous indiquent des voies universelles de recherche qui mèneront certainement à des solutions particulières parfaitement adaptées à des conditions particulières.

\section{L.-René PARENTEAU}

\section{Personnel: The Human Problems of Manage-} ments, Second Edition, by George Strauss and Leonard R. Sayles, Prentice-Hall Inc., New Jersey, 1967, 756 pages.

Par ce «textbook», les outeurs veulent couvrir le spectre complet des questions qui concernent la direction du personnel et les relations humaines.

Le livre indique clairement et de foçon analytique de quelle façon on peut développer des procédures et des politiques appropriées pour en arriver à l'utilisation optimale des ressources humaines. II offre le bagage théorique fondamental des sciences du comportement, y intègre les résultats de la recherche sur les relations humaines, et y ajoute les procédures modernes utilisées dans I'administration des ressources humaines. Le tout est assorti d'études de cas et de problèmes qui veulent initier le lecteur à l'utilisation éventuelle de ces techniques.

Le but des auteurs est de suggérer une approche systématique et unifiée aux problèmes de personnel auxquels font face les organisations industrielles. Les réponses qu'ils fournissent ne sont pas de simples « recettes » (do this or do that). Ils soulignent que dans plusieurs cas, il est très difficile sinon impossible, de même suggérer des réponses. II s'agit plutôt de saisir les a bonnes questions », de bien poser les problèmes.

Cette imposante publication comprend sept (7) parties. On y traite successivement des résultats ou des contributions de diverses spécialités à la compréhension et au règlement des problèmes humains dans l'entreprise.

Les parties I, II et III traitent principalement des fondements psychologiques et sociologiques de la vie à l'intérieur d'une entreprise et à l'intérieur du monde industriel. Partant de l'individu, de ses besoins, de ses aspirations et de son comportement à l'intérieur d'un groupe, on traite successivement 